\title{
Depression and drug utilization in an elderly population
}

\author{
Raffaele Antonelli Incalzi ${ }^{1,4}$ \\ Andrea Corsonello 2,4 \\ Claudio Pedone ${ }^{1,4}$ \\ Francesco Corica ${ }^{3,4}$ \\ Pierugo Carbonin ${ }^{1,4}$ \\ 'Centro di Medicina \\ dell'Invecchiamento, Policlinico A \\ Gemelli, Università Cattolica del \\ Sacro Cuore, Roma, Italy; ${ }^{2}$ Istituto \\ Nazionale di Ricovero e Cura per \\ Anziani (INRCA), Cosenza, Italy; \\ ${ }^{3}$ Dipartimento di Medicina Interna, \\ Università degli Studi di Messina, \\ Messina, Italy; ${ }^{4} \mathrm{On}$ behalf of the \\ Gruppo Italiano di Farmacovigilanza \\ nell'Anziano (GIFA) investigators
}

Correspondence: Andrea Corsonello Via D Frugiuele, 39; I-87I 00 Cosenza, Italy

Tel +3909846821 II

Fax +39098475496

Email andrea_corsonello@tin.it
Objective: To verify whether depression, defined as a 15-item Geriatric Depression Scale $($ GDS $)>6$, is associated with greater drug utilization by elderly patients.

Population: 2568 patients enrolled in the Gruppo Italiano di Farmacovigilanza nell'Anziano (GIFA) study.

Main outcome measure: Polypharmacy, ie, the daily use of at least 3 drugs, excluding antidepressant and anxiolytic agents, in the month prior to admission.

Method: Home therapy data were collected according to a validated procedure. Correlates of polypharmacy were assessed by logistic regression analysis in the whole population and in subgroups for which indexes of disease severity were available.

Results: GDS $>6$ was found to be positively correlated with polypharmacy (odds ratio 1.22; 95\% confidence interval 1.01-1.48) as were older age, comorbidity, hypertension, diabetes mellitus, congestive heart failure, and renal failure. Negative correlates of polypharmacy were smoking habit and alcohol consumption, and GDS $>6$ was negatively associated with the use of analgesic ( $11.8 \%$ vs $15.6 \%, \mathrm{p}=0.012)$. In the subgroups with congestive heart failure and chronic renal failure, GDS $>6$ was strictly associated with greater disease severity, but did not correlate with polypharmacy in multivariable models including indexes of disease severity.

Conclusions: Depressed mood is associated with polypharmacy in the broad elderly population. However, when indexes of disease severity were considered, the association was lost, indicating that depression is a marker of the burden of disease and does not increase drug consumption per se.

Keywords: depression, drugs, elderly, polypharmacy

\section{Introduction}

The elderly are the greatest consumers of drugs in western countries: the daily consumption of drugs rises from $2.0 \pm 1.9$ for people aged $65-74$ years to $2.5 \pm 2.0$ for people aged 75 and over (Chen et al 2001). Polypharmacy has important economic implications and dramatically increases the risk of adverse drug reactions (Carbonin et al 1991; Onder et al 2002; Williams et al 2004). Reducing the number of drugs and rationalizing their utilization, possibly implementing a nonpharmacological approach, is a key component of interventions aimed at improving prescription (Williams et al 2004). Clarifying the determinants of the relationship between age and polypharmacy would be introductory to these kinds of interventions. The age-related increase of concurrent diseases (polypathology) seems the most obvious explanation of the high number of drugs taken by older people, but additional factors deserve consideration. Among these, the perceived need of care could affect the use of drugs, and the finding of a positive association between depression and polypharmacy in an elderly population supports this hypothesis (Simons et al 1992). However, the possibility that depression simply reflects polypathology and/or increases the use of psychotropic drugs only has not been tested. Furthermore, the role of social and cultural factors 
and individual age-related chronic diseases in conditioning the relationship between age and drug use has not been extensively assessed (Simons et al 1992; Al-Windi et al 2004).

The present study aims to identify the main correlates of polypharmacy in a broad elderly population, and to verify whether depression affects drug use per se or as an index of polypathology and poor health status.

\section{Method}

\section{Patients}

The present study uses data from a large observational study group, the Gruppo Italiano di Farmacovigilanza nell'Anziano (GIFA), a collaborative group based in community and university hospitals located throughout Italy. GIFA periodically surveys drug consumption, occurrence of adverse drug reactions, and quality of hospital care. We used data of patients admitted consecutively to the 24 participating centers during the 4-month survey carried out in 1998. Methods of GIFA have been previously described (see Carbonin et al 1991; Carosella et al 1999). Briefly, a study physician with specific training completed a questionnaire for each patient on admission to hospital and updated it daily. Data recorded included sociodemographic characteristics, medical variables, complete blood count, and neuropsychological and physical function variables.

Overall, 3010 patients were enrolled in the survey period. We excluded patients who died during their hospital stay $(n=117)$ and those who were unable to complete the 15 -item Geriatric Depression Scale (GDS) score $(n=325)$, eg, those with severe cognitive deficit. The final sample of patients was 2568 .

The main outcome measure of the study was the daily use of 3 or more drugs prior to admission, where 3 correspond to the 75 th percentile of the number of drugs' distribution. Information on drug therapy in people with cognitive impairment was collected by caregivers. Drugs were coded by Anatomical and Therapeutic Classification (ATC) (Pahor et al 1994).

Variables specifically considered in this study were age, gender, smoking habits, and alcohol consumption. The presence of depressive symptoms was ascertained using the GDS, and patients with a GDS score greater than 6 were considered depressed (Lesher and Berryhill 1994). Functional and cognitive capabilities were measured by the Activities of Daily Living (ADL) scale (Katz et al 1963) and Hodgkinson Abbreviated Mental Test (AMT) (Jitapunkul et al 1991), respectively. Patients having an AMT score of 7 or less were considered cognitively impaired (Rocca et al 1992). Diagnoses were coded according to International Classification of Diseases, Clinical Modification 9th revision (ICD-9 CM) (WHO 1980). Having more than 4 diagnoses was considered as an index of cumulative comorbidity. Furthermore, highly prevalent age-related chronic conditions that may increase the consumption of drugs, such as hypertension, diabetes mellitus, congestive heart failure, cerebrovascular disease, chronic obstructive pulmonary disease, and chronic renal failure were also considered as potential confounders.

Procedures conformed to guidelines provided by the Catholic University Ethical Committee.

\section{Statistical analysis}

Contingency tables were used to compare the demographic and clinical characteristics of patients grouped according to whether they used less than 3 , or 3 or more drugs daily in the month prior to admission. Odds ratios (OR) and the corresponding 95\% confidence intervals (CI), adjusted for age and gender to quantify the association between the use of 3 or more drugs and the variables of interest were used. We then built a multivariable logistic regression model to obtain a deconfounded estimate of the association between depressive symptoms and polypharmacy, adjusting for age, gender and variables proven to be associated with the outcomes in the crude analysis. In a former model, anxiolytic and antidepressant drugs were excluded from the definition of polypharmacy to focus on the utilization of drugs unrelated to depression. Anxiolytics were excluded because of the high prevalence of anxious symptoms in elderly depressed patients (Lenze et al 2001).

One of our objectives was to evaluate whether depression was associated with polypharmacy independent from disease severity. To test this hypothesis, we repeated the analysis described above in people with congestive heart failure as the main disease (ICD-9 codes 428-428.9 and 429.9) using the New York Heart Association (NYHA) class as a marker of disease severity. It was also repeated in people with chronic renal failure as the main disease (ICD-9 codes 580.9, 581.9, 582-583.7, 585, 586, 588-588.9, 593.9), using the Modification of Diet in Renal Disease (MDRD) derived glomerular filtration rate (GFR) (Levey et al 1999) as an index of disease severity. GFR was expressed as $\mathrm{mL} / \mathrm{min} /$ $1.73 \mathrm{~m}^{2}$ body surface area (BSA). BSA was calculated by Mosteller's formula (Mosteller 1987). All analyses were performed using SPSS V10.0 (SPSS Inc, Chicago IL, USA). 
Table I Sociodemographic and clinical characteristics of patients divided according to the number of daily used drugs prior to admission. Antidepressant and anxiolytic drugs were excluded from the computation.

\begin{tabular}{|c|c|c|c|c|c|c|}
\hline \multirow[b]{2}{*}{ Characteristics } & \multicolumn{2}{|c|}{ Used drugs $<3$} & \multicolumn{2}{|c|}{ Used drugs $>3$} & \multirow[b]{2}{*}{$\mathbf{O R}^{\mathrm{a}}$} & \multirow[b]{2}{*}{$95 \% \mathrm{Cl}$} \\
\hline & $\mathbf{N}=979$ & $\overline{(\%)}$ & $\mathbf{N}=1589$ & $(\%)$ & & \\
\hline \multicolumn{7}{|l|}{ Age, years } \\
\hline$<65$ & 406 & 41.5 & 442 & 27.8 & 1.0 & \\
\hline $65-79$ & 351 & 35.9 & 723 & 45.5 & 1.92 & $1.60-2.32$ \\
\hline$>79$ & 222 & 22.7 & 424 & 26.7 & 1.83 & $1.48-2.27$ \\
\hline Gender (Males) & 535 & 54.6 & 910 & 57.3 & 1.20 & $1.02-1.42$ \\
\hline Smokers & 220 & 22.5 & 167 & 10.5 & 0.43 & $0.34-0.54$ \\
\hline Alcohol consumption $>1 / 2 \mathrm{~L}$ & 279 & 28.5 & 357 & 22.5 & 0.68 & $0.56-0.82$ \\
\hline $\mathrm{AMT}<7$ & 169 & 17.3 & 309 & 19.4 & 1.02 & $0.82-1.27$ \\
\hline Dependent in at least I ADL & 77 & 7.9 & 188 & 11.8 & 1.39 & $1.05-1.85$ \\
\hline GDS $>6$ & 245 & 25.0 & 486 & 30.6 & 1.26 & $1.05-1.52$ \\
\hline Having more than 4 diagnoses & 331 & 33.8 & 873 & 54.9 & 2.23 & $\mathrm{I} .88-2.64$ \\
\hline Hypertension & 293 & 29.9 & 690 & 43.4 & $\mathrm{I} .74$ & I.47-2.07 \\
\hline Diabetes & 136 & 13.9 & 368 & 23.2 & 1.85 & |.49-2.31 \\
\hline Congestive heart failure & 66 & 6.7 & 354 & 22.3 & 3.56 & $2.69-4.71$ \\
\hline COPD & 96 & 9.8 & 202 & 12.7 & 1.18 & $0.91-1.53$ \\
\hline Cerebrovascular disease & 129 & 13.2 & 231 & 14.5 & 1.0 & $0.76-1.23$ \\
\hline Chronic renal failure & 30 & 3.1 & $15 \mid$ & 9.5 & 3.02 & $2.02-4.52$ \\
\hline
\end{tabular}

a Adjusted for age and gender.

Abbreviations: OR, odds ratio; $\mathrm{Cl}$, confidence interval; AMT, Hodgkinson Abbreviated Mental Test; ADL, activity of daily living; GDS, Geriatric Depression Scale; COPD, chronic obstructive pulmonary disease.

\section{Results}

Demographic and clinical characteristics of patients divided according to the number of daily used drugs in the last month before the admission are shown in Table 1. Consumers of 3 or more drugs were older and more frequently depressed than consumers of less than 3 drugs. Having more than 4 diagnoses was more frequent among those taking more drugs, as were the diagnoses of hypertension, diabetes mellitus, congestive heart failure, and renal failure. Similar results were obtained including antidepressant and anxiolytic agents in the computation of the number of drugs.

The multivariable logistic regression model showed that a GDS $>6$ is an independent correlate of polypharmacy, together with age, male gender, comorbidity, hypertension, diabetes mellitus, congestive heart failure, and chronic renal failure. Negative correlates of the outcome were smoking habit and alcohol consumption (Table 2). The association between depressive symptoms and polypharmacy was stronger when both antidepressant (ATC codes N06A) and anxiolytic (ATC codes N05B) agents (OR 1.30; 95\% CI 1.07-1.59) were included in the computation of used drugs. When the analysis was limited to patients aged 65 or more, we found a similar association, although with wider CI due to the smaller sample (OR 1.24; 95\% CI 0.96-1.64). To verify whether the relationship between depression and polypharmacy was modified by gender, we used a stratified analysis and calculated the relative odds ratio (ROR) along with its 95\% CI (Altman and Bland 2003). The OR was 1.44 (95\% CI 1.09-1.90) in women and 1.20 (95\% CI 0.89 $1.61)$ in men. The ROR was 1.2 (95\% CI $0.80-1.80)$, indicating no effect modification across gender.

Depressive symptoms were negatively associated with the use of analgesic (ATC codes N02) and/or antiinflammatory (ATC codes M01A) drugs $(16.6 \%$ in depressed patients vs $19.8 \%$ in nondepressed patients, $\mathrm{p}=0.057)$.

Table 2 Summary regression model of selected risk factors to the use of 3 or more drugs, excluded antidepressant and anxiolytic agents, prior to admission

\begin{tabular}{|c|c|c|}
\hline Risk factors & OR & $95 \% \mathrm{Cl}$ \\
\hline \multicolumn{3}{|l|}{ Age, years } \\
\hline$<65$ & 1.0 & \\
\hline $65-79$ & 1.30 & $1.06-1.59$ \\
\hline$>79$ & I.II & $0.98-1.40$ \\
\hline Gender (Males) & 1.50 & $1.24-1.80$ \\
\hline Smokers & 0.42 & $0.33-0.53$ \\
\hline Alcohol consumption $>1 / 2 \mathrm{~L}$ & 0.76 & $0.62-0.93$ \\
\hline Dependent in at least I ADL & 1.33 & $0.98-1.80$ \\
\hline Having more than 4 diagnoses & 1.62 & $1.35-1.95$ \\
\hline Hypertension & 1.58 & $1.35-1.95$ \\
\hline Diabetes & 1.44 & $1.14-1.82$ \\
\hline Congestive heart failure & 3.20 & $2.39-4.29$ \\
\hline Chronic renal failure & 1.90 & $1.24-2.90$ \\
\hline GDS $>6$ & 1.22 & $1.01-1.48$ \\
\hline
\end{tabular}

Abbreviations: $\mathrm{OR}$, odds ratio; $\mathrm{Cl}$, confidence interval; $\mathrm{ADL}$, activity of daily living; GDS, Geriatric Depression Scale. 
In the congestive heart failure (CHF) population $(n=420)$, the prevalence of GDS $>6$ progressively increased through NYHA classes $(I=14.3 \%, I I=32.5 \%, I I I=32.2 \%$, $\mathrm{IV}=66.7 \%$ ). NYHA class (III-IV vs I-II OR $2.75 ; 95 \% \mathrm{CI}$ 1.11-6.83), but not the GDS $>6$ (OR 1.14; 95\% CI $0.73-$ 2.07), was an independent correlate of polypharmacy in this subgroup. In the group with chronic renal failure $(n=181)$, $40 \%$ of patients with GFR $<60 \mathrm{~mL} / \mathrm{min} / 1.73 \mathrm{~m}^{2} \mathrm{BSA}$ had GDS $>6$, compared with none of those having GFR $\geq 60 \mathrm{~mL} /$ $\min / 1.73 \mathrm{~m}^{2}$ BSA. However, the prevalence of severe renal impairment (ie, GFR $<30 \mathrm{~mL} / \mathrm{min} / 1.73 \mathrm{~m}^{2} \mathrm{BSA}$ ) was similar in depressed (39.0\%) and nondepressed (35.8\%) renal failure patients.

In this group, glomerular filtration rate $<30 \mathrm{~mL} / \mathrm{min} /$ $1.73 \mathrm{~m}^{2}$ BSA was associated with polypharmacy (OR 2.79; 95\% CI 0.96-8.06, p=0.059), while GDS $>6$ was not significantly related to the outcome.

In the whole study population, GDS $>6$ was strongly associated with comorbidity $32.7 \%$ in patients having 4 or more diagnoses vs $24.7 \%$ in patients with less than 4 clinical problems, $\mathrm{p}<0.001)$ and physical performance $(46.4 \%$ in patients dependent in at least 1 ADL vs $26.4 \%$ in independent subjects, $\mathrm{p}<0.001)$.

\section{Discussion}

The main finding from our study is that depression is associated with polypharmacy because it is a marker of disease severity and health status impairment. The fact that GDS $>6$ was associated with a lesser consumption of antiinflammatory and/or analgesic drugs suggests that depression per se does not increase the perceived need of care. The consumption of these drugs is considered to depend consistently upon subjective factors, such as the threshold for and the ability to cope with pain, which, in turn, are expected to be influenced by the affective status (Mantyselka et al 2002). This finding is apparently in disagreement with the notion that pain threshold is reduced in patients with depression (Marazziti et al 1998). However, a more recent meta-analysis of 6 independent studies shows that pain perception threshold is higher in depressed subjects (Dickens et al 2003). Furthermore, elderly patients are exposed to the risk of underrecognition and undertreatment of pain because of a mix of cultural and procedural causes (Won et al 1999; Schilling 2003). They may be unable to explain their pain and to ask for care, and physicians have been reported to give less attention to the medical problems of older patients (Radecki et al 1988). All this might explain our results.
Depression was found to be a main correlate of drug consumption in Australian home-dwelling people aged over 59 (Simons et al 1992). However, the study did not assess the relationship between polypharmacy and polypathology or exclude antidepressant drugs. Furthermore, the diagnosis of depression in the Australian study was clinical and not based upon a screening instrument. This is likely to have resulted in the selection of patients more severely depressed than the ones we considered depressed using the GDS. Accordingly, our results add to previous knowledge because they apply to the average elderly population with chronic diseases and stem from a deconfounded estimate of the determinants of polypharmacy.

The findings indirectly confirm that geriatric depression is usually reactive to medical distressing conditions (Hybels et al 2001). Accordingly, a scale testing the affective status can be regarded as a surrogate measure of perceived health (Kennedy et al 2001). On the other hand, measures of cognitive ability and physical dependency were not correlated with the use of drugs, although they are well recognized indexes of health status impairment (Nelson et al 2004). This suggests an alternative explanation to the relationship between consumption of drugs and affective status: polypharmacy might negatively affect the perception of disease status (Fincke et al 1998) and, as a consequence, induce depression. Efforts to simplify the pharmacological therapy might improve the affective status besides having several other beneficial effects, eg, to reduce the risk of adverse drug reactions (Schmader et al 2004; Williams et al 2004).

Alcohol and smoke addiction were associated with a lesser consumption of drugs, and this is consistent with the underreporting of medical problems or with a bias related to the fact that patients in better health are less motivated to correct unhealthy habits. Both these possibilities seem to mediate, for example, the repeatedly reported link between alcohol/smoke and underreporting/undertreatment of hypertension (Gulliford 2001; Corsonello et al 2003).

Surveys on the quality of pharmacological therapy in the elderly have provided conflicting results. Schmader et al (2004) found that a considerable number of curable conditions remained unrecognized and, then uncared for in elderly outpatients, while geriatric evaluation and management are able to reduce the number of conditions with omitted drugs. Williams et al (2004), instead, recognized an excess use of drugs as a relatively common problem; however, they selected only elderly ambulatory patients taking at least 5 drugs and thus obtained results 
that seem poorly representative of the average quality of the pharmacological therapy. In the GIFA study, the use of ACE inhibitors in CHF patients, antihypertensive in hypertensive and statins in hypercholesterolemic patients were found to be very inadequate (Sgadari et al 2000; Antonelli Incalzi et al 2002; Onder et al 2003). Thus, polypharmacy should not be equated to overuse of drugs, rather it frequently means improper use (Gilberg et al 2003; Hanlon et al 2004). Accordingly, efforts to understand factors explaining polypharmacy could improve the quality of drug prescription. Our findings suggest that depression per se is not among the main explanatory factors.

Some limitations of this study should be cited. First, we administered the GDS at the time of patient discharge. Thus, the recorded score might not be fully representative of the affective status prior to admission. Second, an index of disease severity was not available for the whole studied population: this prevented us from testing the hypothesis that GDS $>6$ reflected disease severity. However, the strong relationship of GDS $>6$ to dependency and comorbidity is consistent with this hypothesis. All our patients were hospitalized, and our results might not be generalizable to the outpatients. However, our findings are supported by others (Simons et al 1992) carried out in a noninstitutionalized population. Finally, a cross-sectional observation is exploratory in nature and needs to be validated prospectively.

In conclusion, our data indicate that although depression is significantly related to polypharmacy, it seems not to affect drug use per se, but rather as an index of polypathology and poor health status. Clarifying both the causal link and the determinants of the relationship between depression and polypharmacy could help decrease the burden of both pharmacological therapy and depression in elderly patients. From this perspective, the psychological effects of strategies simplifying pharmacological therapy should be explored. In the same manner, it should be verified whether improving the affective status by nonpharmacological interventions can reduce drug consumption. Further studies would improve our knowledge of the complex dynamics linking perceived health and use of drugs in the elderly.

\section{Acknowledgments}

The Gruppo Italiano di Farmacovigilanza nell'Anziano (GIFA) is a research group of the Italian Society of Gerontology and Geriatrics (SIGG) - Fondazione Italiana per la Ricerca sull'Invecchiamento (FIRI-ONLUS). The GIFA is partially supported by a grant from the Italian
National Research Council (nr 94000402). A complete list of GIFA investigators has been published previously (Eur J Epidemiol, 1999, 15:893-901).

\section{References}

Altman DG, Bland JM. 2003. Interaction revisited: the difference between two estimates. BMJ, 326:219.

Al-Windi A, Elmfeldt D, Svardsudd K. 2004. Determinants of drug utilisation in a Swedish municipality. Pharmacoepidemiol Drug Saf, 13:97-103.

Antonelli Incalzi R, Pedone C, Pahor M, et al. 2002. Trends in prescribing ACE-inhibitors for congestive heart failure in elderly people. Aging Clin Exp Res, 14:516-21.

Carbonin P, Pahor M, Bernabei R, et al. 1991. Is age an independent risk factor of adverse drug reactions in hospitalized medical patients? J Am Geriatr Soc, 39:1093-9.

Carosella L, Pahor M, Pedone C, et al. 1999. Pharmacosurveillance in hospitalized patients in Italy. Study design of the 'Gruppo Italiano di Farmacovigilanza nell'Anziano' (GIFA). Pharmacol Res, 40:287-95.

Chen YF, Dewey ME, Avery AJ; Analysis Group of The MRCCFA Study. The Medical Research Council Cognitive Function and Ageing Study (MRC CFAS). 2001. Self-reported medication use for older people in England and Wales. J Clin Pharm Ther, 26:129-40.

Corsonello A, Antonelli Incalzi R, Pedone C, et al. 2003. Seasonal hypertension: a clue to explain the high prevalence of unrecognized hypertension in the elderly? Gruppo Italiano di Farmacovigilanza nell'Anziano (GIFA). Aging Clin Exp Res, 15:296-300.

Dickens C, McGowan L, Dale S. 2003. Impact of depression on experimental pain perception: a systematic review of the literature with meta-analysis. Psychosom Med, 65:369-75.

Fincke BG, Miller DR, Spiro A III. 1998. The interaction of patient perception of overmedication with drug compliance and side effects. J Gen Intern Med, 13:182-5.

Gilberg K, Laouri M, Wade S, et al. 2003. Analysis of medication use patterns: apparent overuse of antibiotics and underuse of prescription drugs for asthma, depression, and CHF. J Manag Care Pharm, 9: 232-7.

Gulliford MC. 2001. Low rates of detection and treatment of hypertension among current cigarette smokers. J Hum Hypertens, 15:771-3.

Hanlon JT, Artz MB, Pieper CF, et al. 2004. Inappropriate medication use among frail elderly inpatients. Ann Pharmacother, 38:9-14.

Hybels CF, Blazer DG, Pieper CF. 2001. Toward a threshold for subthreshold depression: an analysis of correlates of depression by severity of symptoms using data from an elderly community sample. Gerontologist, 41:357-65.

Jitapunkul S, Pillay I, Ebrahim S. 1991. The abbreviated mental test: its use and validity. Age Ageing, 20:332-6.

Katz S, Ford AB, Moskowitz RW, et al. 1963. Studies of illness in aged: the index of ADL, a standardized measure of biological and psychosocial function. JAMA, 185:94-106.

Kennedy SH, Eisfeld BS, Cooke RG. 2001. Quality of life: an important dimension in assessing the treatment of depression? J Psychiatry Neurosci, 26(Suppl):S23-8.

Lenze EJ, Mulsant BH, Shear MK, et al. 2001. Comorbidity of depression and anxiety disorders in later life. Depress Anxiety, 14:86-93.

Lesher EL, Berryhill JS. 1994. Validation of the Geriatric Depression Scale-Short Form among inpatients. J Clin Psychol, 50:256-60.

Levey AS, Bosch JP, Lewis JB, et al. 1999. A more accurate method to estimate glomerular filtration rate from serum creatinine: a new prediction equation. Modification of Diet in Renal Disease Study Group. Ann Intern Med, 130:461-70.

Mantyselka P, Ahonen R, Viinamaki H, et al. 2002. Drug use by patients visiting primary care physicians due to nonacute musculoskeletal pain. Eur J Pharm Sci, 17:201-6. 
Marazziti D, Castrogiovanni P, Rossi A, et al. 1998. Pain threshold is reduced in depression. Int J Neuropsychopharmcol, 1:45-8.

Mosteller RD. 1987. Simplified calculation of body surface area. $N$ Engl J Med, 317:1098.

Nelson T, Fernandez JL, Livingston G, et al. 2004. Does diagnosis determine delivery? The Islington study of older people's needs and health care costs. Psychol Med, 34:147-55.

Onder G, Gambassi G, Sgadari A, et al. 2003. Impact of hospitalization on blood pressure control in Italy: results from the Italian Group of Pharmacoepidemiology in the Elderly (GIFA). Pharmacotherapy, 23:240-7.

Onder G, Pedone C, Landi F, et al. 2002. Adverse drug reactions as cause of hospital admissions: results from the Italian Group of Pharmacoepidemiology in the Elderly (GIFA). $J$ Am Geriatr Soc, 50:1962-8.

Pahor M, Chrischilles EA, Guralnik JM, et al. 1994. Drug data coding and analysis in epidemiologic studies. Eur J Epidemiol, 10:405-11.

Radecki SE, Kane RL, Solomon DH, et al. 1988. Do physicians spend less time with older patients? J Am Geriatr Soc, 36:713-8.

Rocca WA, Bonaiuto S, Lippi A, et al. 1992. Validation of the Hodkinson Abbreviated Mental Test as a screening instrument for dementia in an Italian population. Neuroepidemiology, 11:288-95.
Schilling ML. 2003. Pain management in older adults. Curr Psychiatry Rep , 5:55-61.

Schmader KE, Hanlon JT, Pieper CF, et al. 2004. Effects of geriatric evaluation and management on adverse drug reactions and suboptimal prescribing in the frail elderly. Am J Med, 116:394-401.

Sgadari A, Incalzi RA, Onder G, et al. 2000. Lipid-lowering therapy in patients with coronary artery disease: sex or age bias? Arch Intern Med, 160:2684-5.

Simons LA, Tett S, Simons J, et al. 1992. Multiple medication use in the elderly. Use of prescription and non-prescription drugs in an Australian community setting. Med J Aust, 157:242-6.

Williams ME, Pulliam CC, Hunter R, et al. 2004. The short-term effect of interdisciplinary medication review on function and cost in ambulatory elderly people. J Am Geriatr Soc, 52:93-8.

Won A, Lapane K, Gambassi G, et al. 1999. Correlates and management of nonmalignant pain in the nursing home. SAGE Study Group. Systematic Assessment of Geriatric drug use via Epidemiology. $J$ Am Geriatr Soc, 47:936-42.

World Health Organization. 1980. PHS-HCF. International classification of diseases, 9th revision. Clinical modifications. Public Health Service-Health Care Financing Administration, Washington. 